\title{
Hausarzt in der Notfallpraxis des Zentrumspitals
}

Rudolf Horn,

Vladimir Sibalic,

Joseph Osterwalder

1 Kissling B. Forum klinische Notfallmedizin fKNM SGNOR. PrimaryCare. 2007;7:12.

2 Gnädinger M, Doenecke C, Chlibec T, Hartmann A, Pfister M. Nächtliche Abdeckung des hausärztlichen Notfalldienstes durch das Akutspital. PrimaryCare. 2009;9:12-3.

3 Triaca H, Ritschard T. Das Notfalldienstmodell im Ärztenotfallkreis Lyss und Umgebung - Spital Aarberg. PrimaryCare. 2007;7:23.

*Zugunsten der Leserlichkeit wird auf die Erwähnung der weiblichen Form verzichtet.

Korrespondenz: PD Dr. Joseph Osterwalder Kantonsspital St. Gallen Rorschacherstrasse 95 CH-9007 St. Gallen Tel. 0714941111

\section{Probleme und Lösungsmöglichkeiten in der Notfallversorgung}

Sowohl in ländlichen wie in städtischen Regionen wird es zunehmend schwierig, die hausärztlichen Notfalldienste rund um die Uhr zu gewährleisten. Die Gründe dafür sind vielfältig. Gemäss Gesetz sind die Kantone für die Organisation der Notfalldienste zuständig. In aller Regel wird die Planung an die regionalen Ärztegesellschaften delegiert. Dies ist insofern auch sinnvoll, da z. B. im Kanton St. Gallen die Probleme und somit Lösungsansätze im Toggenburg und in der Stadt St. Gallen vollkommen unterschiedlich sind. Einige typische Beispiele werden im Forum klinische Notfallmedizin SGNOR [1] erwähnt. So wird z. B. im Spital Rorschach [2] oder Notfallkreis Lyss [3] das Notfalltelefon nachts in das Spital umgeleitet. Auf der Infoplattform Notfalldienst der Gesundheitsdirektorenkonferenz werden viele der gängigen Modelle beschrieben (www.gdk-cds.ch/test.0.html).

\section{Zusammenarbeit der Hausärzte der Stadt St. Gallen mit dem Kantonsspital St. Gallen}

\section{Ausgangslage}

In der Zentralen Notfallaufnahme des Kantonsspitals St. Gallen werden mehr als 30000 Patienten* pro Jahr betreut. Seit Jahren steigt sowohl die Zahl der ärztlichen Zuweisungen als auch diejenige der Selbstzuweisungen stetig an. Viele der «Walk-in-Patienten» benötigen keine spezialärztliche Behandlung und müssen nicht hospitalisiert werden. Anderseits nahm in letzter Zeit die Anzahl der Notfallpatienten im stadtärztlichen Notfalldienst ab. 50 bis 60 Ärzte leisteten den Notfalldienst in der Stadt St. Gallen sowie der Gemeinden Abtwil, Engelburg, St. Josefen, Häggenschwil, Kronbühl, Lömmenschwil und Wittenbach. Auch hier wurde es zunehmend schwierig, Ärzte für den Notfalldienst zu gewinnen.

\section{Lösung}

Die Ärzte des Kantonsspitals und die Hausärzte entschlossen sich zu einer Zusammenarbeit mit dem Ziel, dass einerseits die Zentrale Notfallaufnahme (ZNA) entlastet und anderseits die Situation der Hausärzte verbessert wird. Dazu wurde die Zentrale Notfallaufnahme in einen Kliniknotfall (ZNA II) und eine Notfallpraxis (= Grundversorgernotfall ZNA I) aufgeteilt. In der ZNA I behandeln tagsüber erfahrene, breit ausgebildete Oberärzte Selbstzuweiser, die nicht hospitalisiert werden müssen und keine spezialärztliche Behandlung benötigen. Am Abend, von 17-23 Uhr,

\section{La médecine de famille aux urgences d'un centre hospitalier}

A Saint-Gall, le service centralisé des urgences a été divisé en une clinique pour les urgences (ZNA II) et un cabinet de garde (= urgences pour les soins de premiers recours ZNA I). La journée, dans le service ZNA I, des médecins urgentistes, expérimentés et au bénéfice d'une formation approfondie, prennent en charge les patients ambulatoires qui se présentent directement à l'hôpital avec de petits problèmes de santé. Le soir et le week-end, tout le service d'urgence en charge des soins de premier recours de la ville de Saint-Gall et de ses environs est assuré par des médecins de famille dans le service ZNA I. Par ailleurs, un médecin supplémentaire est disponible $\mathbf{2 4}$ heures sur $\mathbf{2 4}$ pour les visites à domicile. La nuit, les médecins internistes de garde répondent aux appels d'urgences pour conseiller les patients sur toute question relative à la médecine de famille. Après un an, une évaluation montre que pratiquement toutes les personnes impliquées affichent une satisfaction supérieure à la moyenne quant à leur nouveau système de prise en charge des soins d'urgence.

sowie am Wochenende 10-23 Uhr, arbeitet ein Hausarzt in der ZNA I. Er behandelt sowohl die Selbstzuweiser als auch die Patienten, die sich über das hausärztliche Notfalltelefon anmelden. Das Notfalltelefon bedient der diensthabende Hausarzt von 7-23 Uhr. Von 23-7 Uhr wird es dem Dienstarzt der Inneren Medizin übergeben, der die Patienten bei Bedarf ins Spital bestellt. Die Telefonate müssen von den Dienstärzten schriftlich festgehalten werden, so dass Unklarheiten am Folgetag diskutiert werden können. Neben dem Notfalldienst in der ZNA I ist während 24 Stunden am Tag ein weiterer Hausarzt für Hausbesuche abrufbereit. Die im Spital diensttuenden Hausärzte werden im Stundenlohn entschädigt, das Spital stellt die Rechnungen. Nach zwei Pilotprojektphasen wurde die Zusammenarbeit in beschriebener Form am 1.4.2009 definitiv gestartet. 


\section{Die Statistik des ersten Jahres}

Insgesamt wurden im ersten Jahr der Zusammenarbeit (1.4.2009-31.3.2010) von Hausärzten und dem Kantonsspital St. Gallen 6931 Patienten in der Notfallpraxis ZNA I behandelt. $1 / 3$ der Patienten wurde durch die Spitalärzte, $2 / 3$ durch die Hausärzte gesehen. Knapp die Hälfte waren Unfallpatienten. Bei 3\% der Patienten wurden die Spezialarztkollegen um Mitbeurteilung gefragt. $5 \%$ der Patienten, die vorerst durch den Grundversorger gesehen wurden, mussten hospi-

\section{Abbildung 1}

VAS 1-10 (visual analog scale); 1 = Arbeitsbelastung sehr klein, $10=$ Arbeitsbelastung sehr gross.

\section{Wie ist die Belastung bei der Arbeit in der ZNA I}

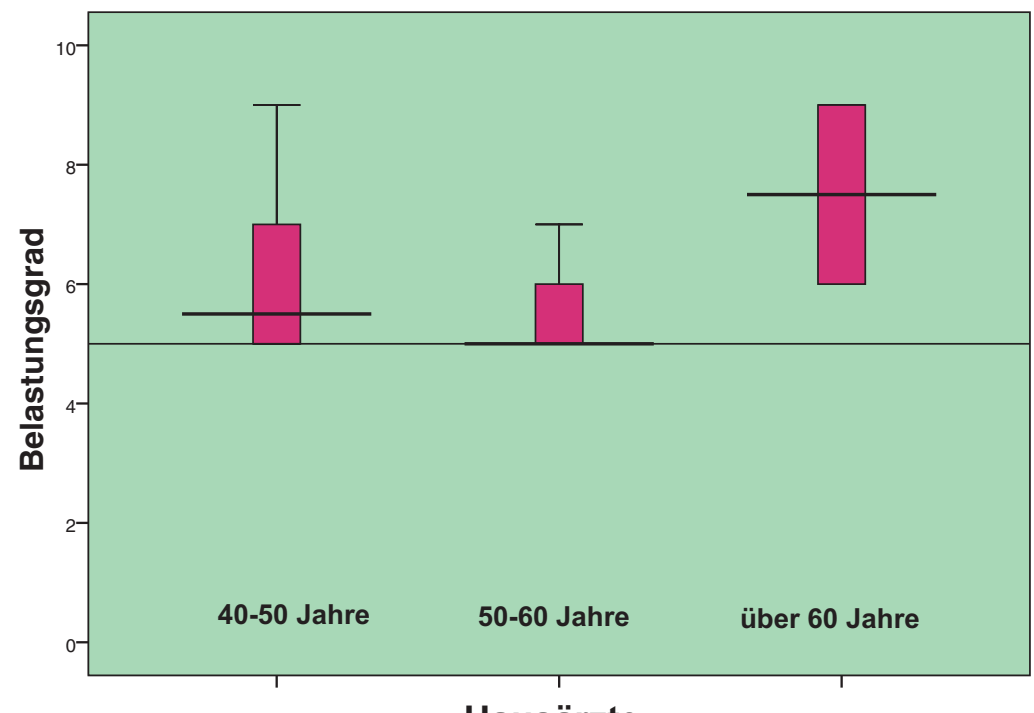

Hausärzte

Abbildung2

VAS 1-10 (visual analog scale); 1 = überhaupt nicht zufrieden, $10=$ sehr zufrieden.

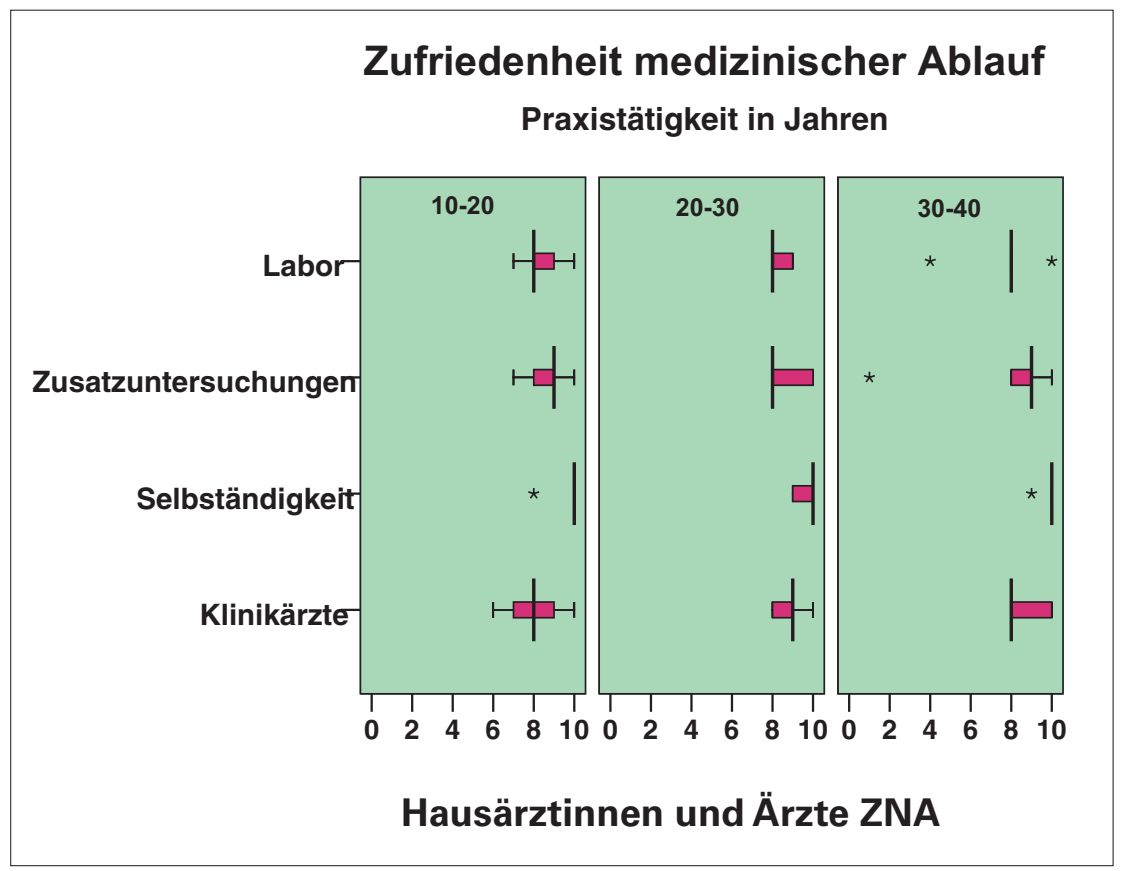

talisiert werden (Übergabe in ZNA II). An den Wochenenden gab es deutlich mehr Patienten als während der Woche. Die durchschnittlichen Aufenthaltszeiten für die Patienten mit einfachen Notfällen konnten deutlich reduziert werden (von 2,5 Stunden auf 1,2 Stunden).

\section{Evaluation}

\section{Gründe}

Einerseits wollten wir die Zufriedenheit der Mitarbeitenden im neuen Notfalldienstmodell systematisch erfassen. Anderseits hofften wir, auf diese Art Ideen zur Verbesserung der Organisation zu bekommen.

\section{Methode}

Folgende Gruppen von Mitarbeitern wurden befragt: Hausärzte, in der ZNA I tätig; Hausbesuchsärzte; Klinikärzte: Assistenzärzte und Oberärzte; Dienstärzte Innere Medizin (betreffend Notfalltelefon nachts); Ärztliche Teamleader (Oberärzte) der ZNA; Schichtleitung Pflege; Medizinische Praxisassistentinnen (= Pflege ZNA I). Zusätzlich befragten wir 120 Patienten. Die präzis gestellten Fragen wurden anhand einer VASSkala (visual analog scale, 1-10) beantwortet. Die Fragebogen waren anonym, lediglich mit Angabe der betreffenden Berufsgruppe. Für die Auswertungen verwendeten wir das SPSS-Programm, die Graphiken wurden als Woxblot erstellt.

\section{Hausärzte, die in der ZNA I den Notfalldienst} absolvieren

- Die Hausärzte sind zu 68\% Allgemein-Mediziner und zu $32 \%$ Internisten.

- Hausärzte, die älter als 60 Jahre sind, empfinden den Dienst deutlich belastender (VAS 7/10) als die jüngeren (VAS 5/10) (Abb. 1).

- Die Selbständigkeit bei der Arbeit war gewährleistet (VAS 9/10). Dies war einer der meistdiskutierten resp. befürchteten Punkte bei den Hausärzten (Abb. 2).

- Der medizinische Ablauf mit den Zusatzuntersuchungen und Konsilien wurde fast durchwegs gelobt (VAS 8-9/10) (Abb. 2).

- Die Arbeit in der ZNA wurde von den meisten Ärzten als bereichernd beurteilt (VAS 8/10).

- Seitdem die Hausärzte in der ZNA tätig sind, hat sich die Zusammenarbeit Hausarzt-Spitalarzt verbessert (VAS 7/10 bei den Ärztinnen, VAS 8/10 bei den Ärzten).

\section{Hausbesuchsärzte}

- Die Indikationen für einen Hausbesuch wurden meistens korrekt gestellt (VAS 9/10), dies sowohl bei den Hausärzten wie auch bei den Dienstärzten Innere Medizin.

- Die Arbeitsbelastung, obwohl sie nach dem neuen System für die ganze Stadt zuständig sind, ist gering (an Wochentagen etwas tiefer als an Wochenenden). 
Klinikärzte (Assistenzärzte und Oberärzte)

- Die älteren Kollegen fanden die bei den Hausärzten durchgeführten Konsilien fachlich korrekt (VAS 6/10), die jüngeren eher weniger (VAS 4/10).

- Die Zusammenarbeit auf menschlicher Ebene zwischen den Klinikärzten und den Hausärzten wird insgesamt sehr gelobt.

Abbildung 3

Beurteilung des Einsatzes am hausärztlichen Notfalltelefon.

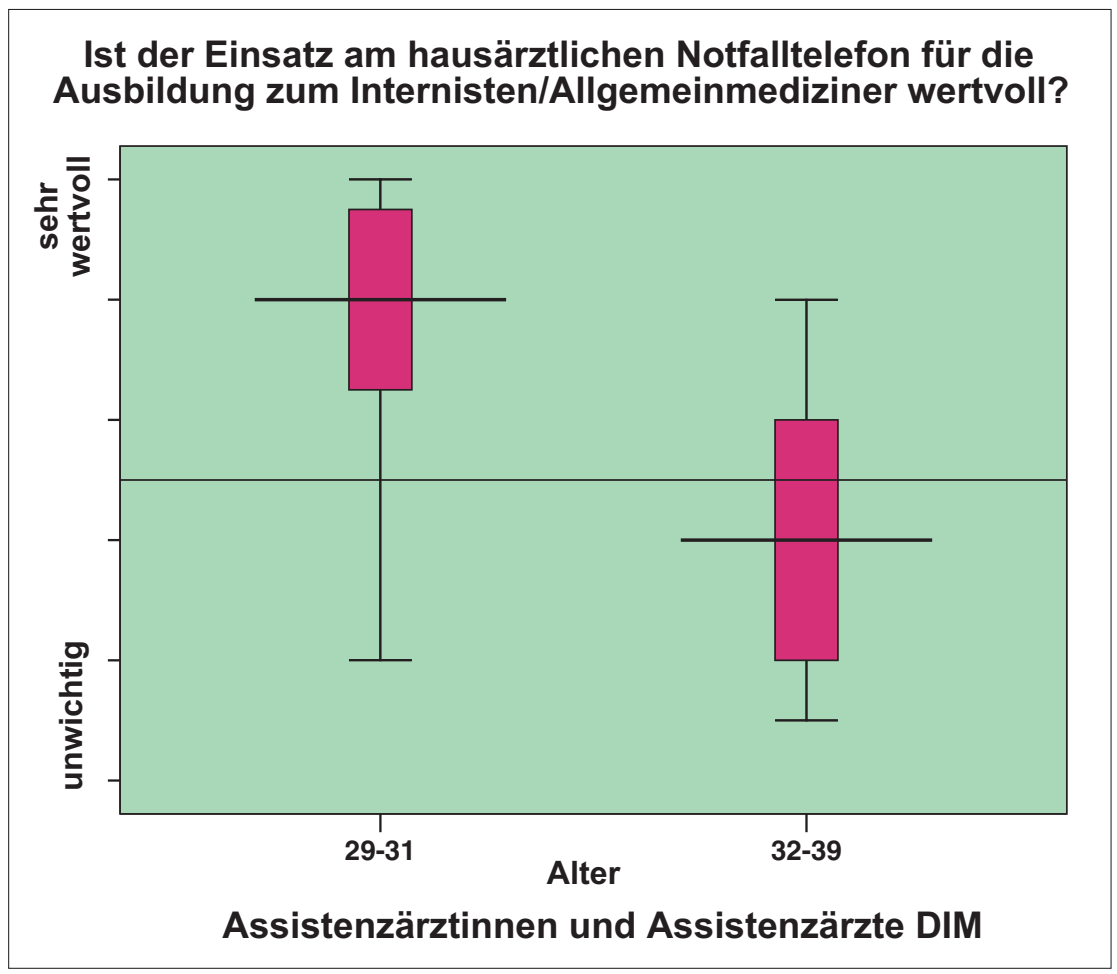

Abbildung 4

Beurteilung der Patienten zum Einsatzort des Notfallarztes.

\section{Wo finden Sie es besser, dass der Notfallarzt arbeitet, im Spital oder in seiner Praxis?}

\section{Alter Patienten}

bis 39 Jahre

über 40 Jahre
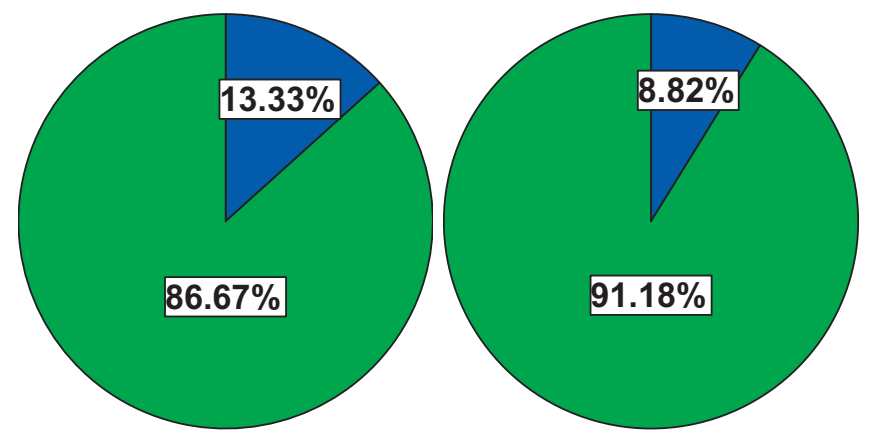

- Kritisiert wird, dass die Assistenzarztausbildung bei einfachen Notfällen jetzt etwas zu kurz kommt.

- Die Übergabe von Patienten, die hospitalisiert werden müssen, ist teilweise noch verbesserungsbedürftig.

Dienstärzte Innere Medizin betreffend nächtliches Notfalltelefon

- Im Median gibt es einen Telefonanruf pro Nacht. Die Belastung ist sowohl in fachlicher wie in zeitlicher Hinsicht nicht besonders hoch. Problematisch ist (wie immer auf einer Notfallstation), dass häufig gleichzeitig ein akut gefährdeter Patient zu behandeln ist und ein hausärztliches Beratungsgespräch am Telefon stattfinden sollte.

- Die jüngeren Kollegen empfinden den Einsatz am hausärztlichen Notfalltelefon für die Ausbildung wertvoll, die älteren Kollegen eher unwichtig (Abb. 3).

\section{Pflegedienst}

- Die Zusammenarbeit mit den Hausärzten empfinden die Mitarbeitenden als sehr angenehm und häufig bereichernd.

\section{Patienten}

- $\quad 87 \%$ der jüngeren Patienten finden es besser, wenn der Hausarzt im Spital statt in seiner Praxis Notfalldienst leistet. Von den älteren Patienten sind dies sogar 91\% (Abb. 4). Dies hängt möglicherweise damit zusammen, dass sich gewisse Hausarztpraxen in einer oberen Etage ohne Lift befinden. Auch muss die Praxis bei ortsunkundigen Patienten zuerst gesucht werden. Des weiteren gibt es viele Hausärzte, die weder röntgen noch Unfälle behandeln (gipsen, Wunden nähen usw.). Die Notfallbehandlung im Spital hat gewisse Vorteile, weil alle Untersuchungen vor Ort möglich sind und der Patient trotzdem von einem breit ausgebildeten Hausarzt angeschaut und beurteilt wird.

\section{Schlussfolgerung}

Es handelt sich um eine Win-Win-Situation: Mit der oben beschriebenen Organisation wurde die Zusammenarbeit zwischen den Hausärzten und Spitalärzten verbessert. Ressourcen konnten eingespart werden und die ZNA II wurde entlastet. Die Aufenthaltszeiten für die Patienten mit einfachen Notfällen wurden deutlich reduziert. Durch die Tätigkeit im Spital bietet sich für die Hausärzte die Möglichkeit, gewisse Krankheiten/Unfälle mit den Spezialärzten zu diskutieren. Am Abend und Wochenende müssen keine MPAs beschäftigt werden, und um $23.00 \mathrm{Uhr}$ ist Dienstschluss. Die Hausbesuchsärzte haben zwar einen 24-Stunden-Dienst; infolge der Vorselektion durch den Hausarzt ZNA I resp. den Dienstarzt Innere Medizin werden sie jedoch deutlich weniger gestört. 